\title{
Efficacy of Posterior Short-Segment Pedicle Fixation in Management of Incomplete Thoracolumbar Burst Fractures without Neurological Impairment
}

\author{
Abdusattarov Khurshid*, Alimov Ijod, Khujanazarov Ilkhom, Karimov Murod and Alikhodjaeva Gulnara \\ Department of Traumatology, Orthopedics, Military field Surgery and Neurosurgery, Tashkent Medical Academy, Uzbekistan
}

Submission: November 13, 2020; Published: December 07, 2020

*Corresponding author: Abdusattarov Khurshid, Department of Traumatology, Orthopedics, Military field Surgery and Neurosurgery, Tashkent Medical Academy, Tashkent, Uzbekistan

\begin{abstract}
Objectives: To evaluate short- and medium-term radiological and clinical outcomes of patients with incomplete thoracolumbar burst fractures (McCormack load-sharing (LSC) score $\leq 6$ ) without neurological deficit who underwent conventional short-segment posterior fixation (SSPF).

Methods: A retrospective study, the radiological and clinical data of patients with a single-level incomplete thoracolumbar burst fractures who admitted SSPF were retrospectively reviewed from 2017-2018. All variables were analysed using descriptive statistical methods.The nonparametric Mann-Whitney U tests used to infer the significance of the statistical difference between preoperative, postoperative and 12-month follow-up outcomes.

Results: A total number of 66 patients, the mean preoperative LSC score was $4.5 \pm 1.3$ points. The mean pre-operative $\mathrm{SI}^{\circ}$ was $14.9 \pm 3.6^{\circ}, 1$ week after surgery the mean $\mathrm{SI}^{\circ}$ was $4.8 \pm 3.3^{\circ}$; at 6 and 12 months follow-up the mean $\mathrm{SI}^{\circ}$ were $5.2 \pm 2.1^{\circ}$ and $5.4 \pm 2.5^{\circ}$, respectively (p<0.001). The computation of the $\mathrm{ABHC} \%$ revealed that the mean preoperative $\mathrm{ABHC} \%$ was $32.8 \% \pm 9.2 \%, 1$-week postoperatively the mean $\mathrm{ABHC} \%$ was $11.6 \% \pm 3.4 \%$, at 6 month follow-up the mean ABHC \% was $11.2 \% \pm 2.3 \%$, whereas at 12 month follow-up the mean ABHC $\%$ was $12.1 \% \pm 2.4$ $\%(\mathrm{p}<0.001)$. The mean VAS score of back pain was improved from preoperative $7.4 \pm 1.3$ to postoperative $2.3 \pm 1.2$, at 6 - and 12 -month follow-up assessments were $1.2 \pm 0.6$ and $1.2 \pm 0.4$ respectively $(\mathrm{p}<0.001$ ). There was no case of implant failure.
\end{abstract}

Conclusion: The results of this study demonstrated that SSPF of a single-level traumatic incomplete thoracolumbar fracture (preoperative LSC score $\leq 6$ ) provided favourable radiological and clinical outcomes at 12-months follow-up, nevertheless patients are required the long- term observation after surgery.

Keywords: Incomplete thoracolumbar burst fractures; McCormack load-sharing (LSC) score; Posterior short-segment pedicle screw fixation

\section{Introduction}

The incidence of thoracolumbar fracture has increased globally, the predictive economic model of "The Global spinal implants and surgery devices market" is forecasted that the budget expenditure will be significantly increased from USD 10.3 billion in 2019 to USD 13.8 billion in 2025 [1]. Despite usage of advanced technologies and surgical techniques in spinal surgery, most patients with thoracolumbar burst fractures have significant disability. The burst fractures of the thoracolumbar spine represent $50 \%$ of all thoracolumbar injuries, of which $21 \%$ of those are incomplete burst fractures [2]. According to the updated AOSpine injury classification system, the Type A3 fracture represents an incomplete burst fracture, which is defined as an injury of the single endplate, superior or inferior, with 2 the involvement of the posterior vertebral wall [2,3]. Almost $50 \%$ of the patients with incomplete thoracolumbar burst fractures are without neurological impairment [4].

The surgical management of incomplete thoracolumbar burst fractures in neurologically intact patients offers immediate stability, decompression of the spinal canal, earlier ambulation and sooner return to daily activities $[5,6]$. The surgical approaches to the management of thoracolumbar burst fractures include anterior decompression and fusion, posterior instrumentation 
with or without decompression, and combined anterior-posterior approach; those modalities can be performed by traditional open surgery or minimally invasive techniques [7]. One of the surgical techniques is conventional short segment pedicle screw fixation (SSPF), which offers the following advantages: straightforward approach and sufficient stabilization, preservation of two or more vertebral motion segments by avoiding unnecessary fusion in the mobile healthy spinal segment, decrease surgical time and operative blood los; those factors can contribute lower morbidity and faster mobilization of the patients. Nevertheless, the risk of postoperative complications such as instrumentation failure, loss of reduction, subsequent kyphosis was reported in clinical practice $[8,9]$. Therefore, our study was designed to evaluate the short- and medium-term radiological and clinical outcomes of patients with incomplete thoracolumbar burst fractures (McCormack load-sharing (LSC)score $\leq 6$ ) without neurological deficit who underwent conventional SSPF with polyaxial pedicle screws.

\section{Material and Methods}

Data were collected retrospectively from the medical records of patients treated between 2017 and 2018 at the department of "Traumatology, orthopedics, neurosurgery with military surgery "of the Tashkent Medical Academy (TMA). The study was executed in compliance with the Helsinki Declaration and local data protection regulations of the TMA. The database included data from patients who signed an informed consent form allowing the use of their clinical and radiological data for research purposes; the anonymous database was generated for the statistical analysis.

The inclusion criteria of patients to this study were: the time interval between injury and surgical treatment of $<72$ hours, single-level incomplete burst fracture (AOSpine Type A3 fracture), both of the vertebral pedicles are intact without any defect on the cortical wall, the level of fractures (T11-L2) without neurologic impairment, McCormack load-sharing (LSC) score $\leq 6$, patients who underwent posterior SSPF (1 level above and 1 below the fracture level) without fusion, age of patients were ranging from 25 to 50 years at the time of injury, the minimum follow-up of patients was 12 months. The exclusion criteria of patients were: osteoporosis (BMD $T$ score $\leq 2.5 \mathrm{SD}$ ), body mass index (BMI) is $\geq 35 \%$, chronic musculoskeletal disease, specific spinal disorders and deformities, pathologic fractures due to the malignancies or osteoporosis, spinal cord injury, previous thoracolumbar spine surgery and polytrauma. The accurate selection of patients was performed according to the inclusion and exclusion criteria, afterward, a total of 66 patients were enrolled in this study. The following variables were retrieved from medical records of patients: demographics characteristics, mechanism of injury, the time interval between trauma and surgery, level of fractures, radiological parameters, surgical and clinical outcomes, duration of hospitalization and complications.
Demographic parameters such as the age of the patients at the time of index procedure, gender, and body mass index (BMI) were included. Radiological and clinical outcomes were evaluated before surgery, 1 week after surgery, at 6- and 12-month followups. Imaging assessment was performed by multi-slice computed tomography and $\mathrm{x}$-ray. The radiological evaluation of fractures included the measurements of the sagittal index $\left(\mathrm{SI}^{\circ}\right)$ and the percentage of anterior body height compression (ABHC \%). The $\mathrm{SI}^{\circ}$ was calculated with the following formula: $\mathrm{SI}^{\circ}=\mathrm{Cobb}$ angle of kyphosis - angle of normal vertebra, where the Cobb angle of kyphosis was the angle between lower endplates of injury vertebral and upper normal vertebral, and angle of normal vertebra is a baseline sagittal curve at the level of the fracture $\left(5^{\circ}\right.$ in thoracic spine segments ( T10-T11), $0^{\circ}$ at the thoracolumbar junction (T12-L1), and $-10^{\circ}$ in the lumbar spine (L2-L3).The $\mathrm{ABHC} \%$ is calculated by the formula: $\mathrm{ABHC} \%=100-2 \mathrm{a} /(\mathrm{b}+\mathrm{c})$ 100 , where $a-$ the height of fractured vertebra, $b$ - the height of the proximal vertebra, and c - the height of the distal vertebra.

Clinical outcome parameters were composed of the Visual Analogue Scale (VAS) score, intraoperative blood loss, duration of the surgery, perioperative and postoperative complications. Upon considering the VAS score: $0=$ absence of pain, $2=$ mild pain, $4=$ moderate pain, $6=$ severe pain, $8=$ extreme pain $10=$ worst pain.

Statistical analysis was performed using SPSS for Windows version 18.0 (SPSS Inc., Chicago, IL, USA). All variables were analysed using descriptive statistical methods. Categorical variables are expressed as frequencies and percentages. Continuous variables are presented as the mean and standard deviation (SD). The non-parametric Mann-Whitney U tests used to infer the significance of the statistical difference between preoperative, postoperative and follow-up outcomes. Statistical significance level equal to 0.05 was used for pairwise comparison of means. The differences were considered statistically significant when $\mathrm{p}<0.05$.

\section{Results}

Between 2017 and 2018, a total of 66 patients with incomplete thoracolumbar burst fractures (T11-L2) without neurological impairment underwent SSPF, of them there were 48 male and 18 female patients. Most injuries resulted from falls from height (71\%), traffic accidents (18.4\%) and sports accidents (10.6\%). The mean time interval between injury and admission to surgery was $44.3 \pm 17.2$ hours. The distribution of the injuries by vertebral segments was the following: the most commonly at the level of L 1 (67.22\%), then Th12 (26.11\%) and L2 (16.46\%). The mean preoperative LSC score was $4.5 \pm 1.3$ points. The mean age of patients at the time of the surgery was $41.76 \pm 8.5$ years, while the mean BMI was $25.8 \pm 4.67\left(\mathrm{~kg} / \mathrm{m}^{2}\right)$.The radiological evaluation of $\mathrm{SI}^{\circ}$ demonstrated that the mean pre-operative $\mathrm{SI}^{\circ}$ was $14.9 \pm 3.6$ $\circ$, while after 1 week following the operation the results showed 
that the significant reduction of the mean $\mathrm{SI}^{\circ}$, which was $4.8 \pm$ $3.3^{\circ}$. The long-term postoperative follow up demonstrated the following results: at 6 months follow-up, the mean $\mathrm{SI}^{\circ}$ was $5.2 \pm$ $2.1^{\circ}$, whereas at 12 months follow-up the mean $\mathrm{SI}^{\circ}$ was $5.4 \pm 2.5$ $\circ$. There were significant statistical differences in the mean of $\mathrm{SI}^{\circ}$ between preoperative, postoperative and follow-up outcomes (all $\mathrm{p}$ - values $<0.001$, statistical significance level $\alpha=0.05$ ) (Figure 1 ).

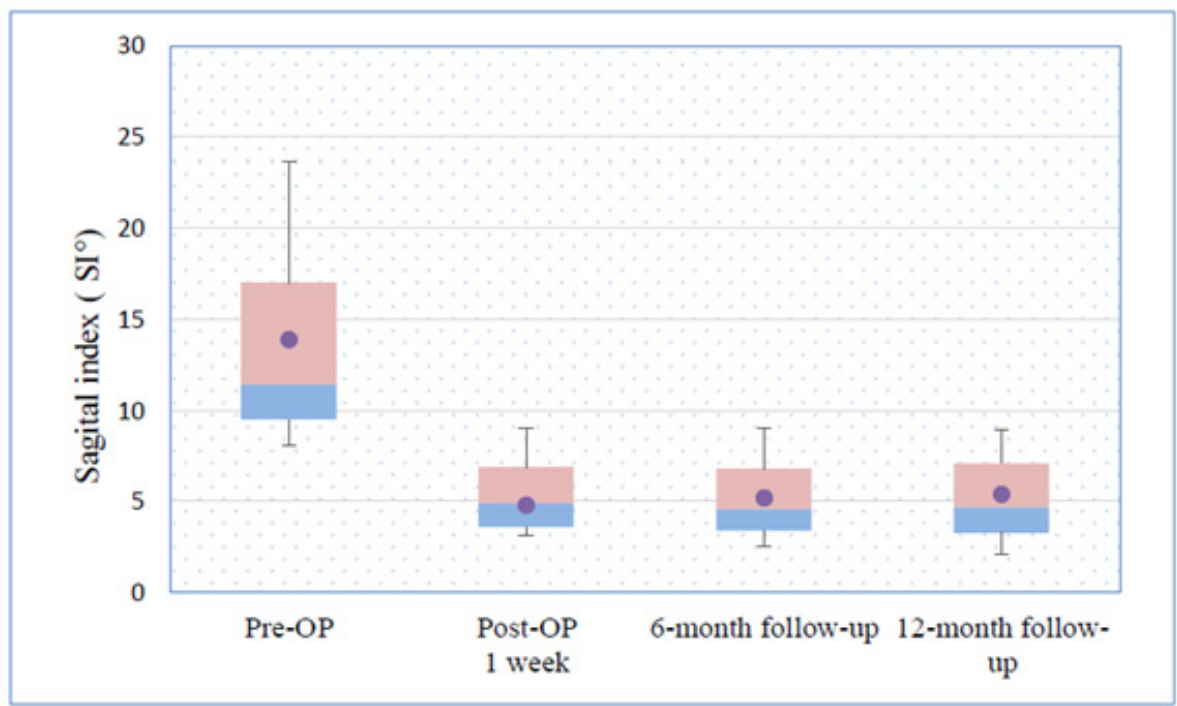

Figure 1: Assessment of Sagital Index angle before and after short segment posterior fixation with pedicle screws.

The estimation of $\mathrm{ABHC} \%$ revealed that the mean preoperative ABHC $\%$ was $32.8 \% \pm 9.2 \%$, whereas the 1 -week postoperatively the mean $\mathrm{ABHC} \%$ was $11.6 \% \pm 3.4 \%$, at 6 months follow-up the mean $\mathrm{ABHC} \%$ was $11.2 \% \pm 2.3 \%$, whereas the subsequent at 12 months follow-up the mean $\mathrm{ABHC} \%$ was $12.1 \% \pm 2.4 \%$. There were significant statistical differences in mean of ABHC $\%$ between preoperative, postoperative follow-up outcomes (all p-values $<0.001$, statistical significance level $\alpha=0.05$ ) (Figure 2).

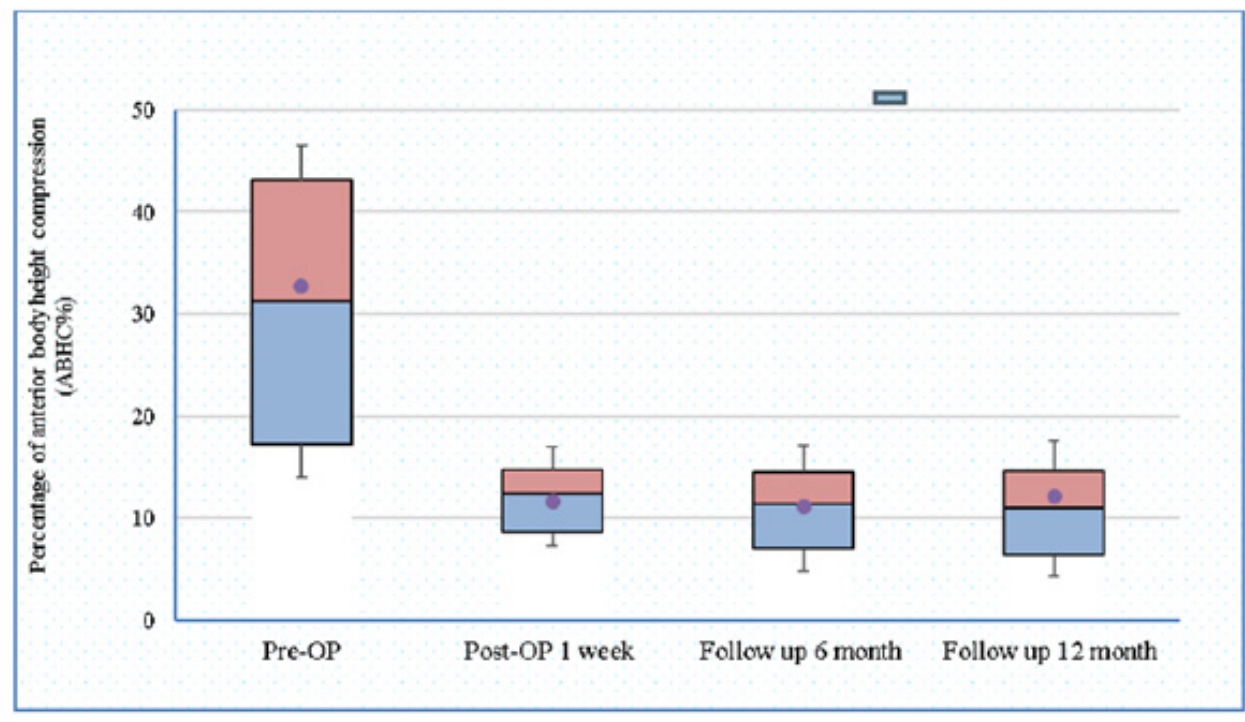

Figure 2: Differences of $\mathrm{ABHC} \%$ prior and after the short segment posterior fixation with the pedicle screws. 
The mean preoperative VAS score was $7.4 \pm 1.3$, whilst at 1 -week after surgery, the VAS score was declined up to the mean $2.3 \pm 1.2$. The assessment of long-term outcomes of the VAS score revealed that at a 6-month follow-up the VAS score was $1.2 \pm 0.6$, and at 12 -month follow-up the mean VAS score was $1.2 \pm 0.4$. The preoperative and postoperative follow-ups (1-week, 6 and 12 months) assessment of the mean VAS scores were statistically significantly different (all $p$ - values $<0.001$, statistical significance level $\alpha=0.05$ ) (Figure 3).

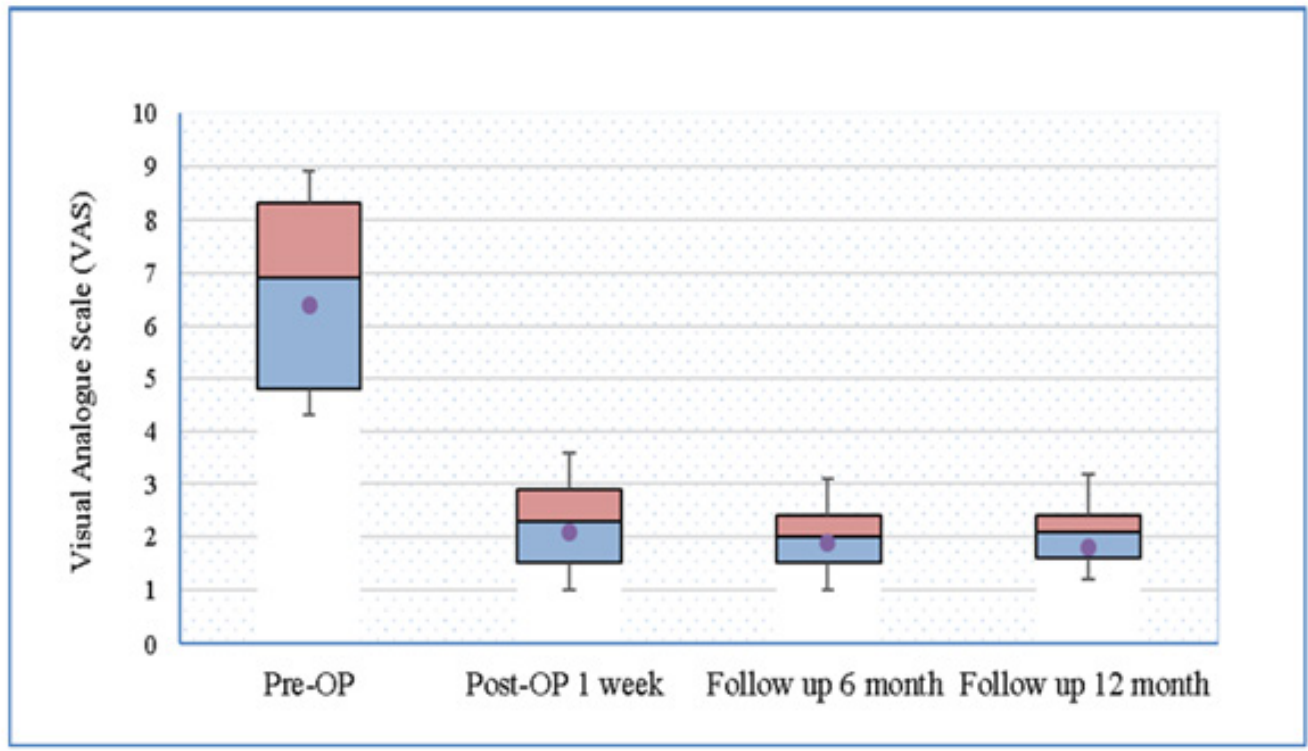

Figure 3: Assessment of the pain outcome using Visual analog scale (VAS) prior and after short segment posterior fixation with the pedicle screws.

The mean duration of the operation was $112.4 \pm 28.2(\mathrm{~min})$ and the mean amount of blood loss was $236 \pm 101(\mathrm{~mL})$. There was no case of implant failure, as well as other complications were not observed. The mean length of hospital stay was $14.5 \pm 3.2$ days. The average follow-up time was $14.3 \pm 2.2$ months.

\section{Discussion}

To date, there are several controversial challenges in the treatment of AO Type A3\& A4 fractures in neurologically intact patients; one of the main issues is the selection of the optimal surgical approach with the minimal risk of complications. The AOSpine thoracolumbar injury classification system \& McCormack load-sharing classification was used in this study to determine the surgical approach for the treatment AO Type A3 fractures. In thus study, the posterior approach has been advocated for all patients with AO Type A3 fractures, when the average preoperative LSC score was $4.5 \pm 1.3$ points. The posterior approach can be effective for the treatment AO Type A3 fractures when LSC score $\leq 6$ points, whereas the anterior approach with anterior reconstruction should be recommended for fractures with LSC score $\geq 6$ points [10]. Tan et al. 2017 conducted a systematic review 6 and metaanalysis, which was evaluated the differences between anterior and posterior approaches in the treatment of AO Type A3 \& A4 fractures. The results of this study demonstrated that neurological, radiological, and functional outcomes were no differences between the two approaches; but the length of hospital stay, and estimated blood loss were higher in the anterior approach [11].

Another important issue is the selection optimal fixation model for the management of AO Type A3 fractures, which can achieve and maintain stabilization in long-term vision. Currently, the most used surgical procedures for the treatment AO Type A3 fractures are mono-segment fixation (MSF) or bi-segmental pedicle fixation [SSPF/SSPF+ intermediate screw (IS) at the fracture level]. The relative advantages and disadvantages of SSPF, SSPF+IS, and MSF for the management of AO Type A3 fractures are conflicting [12-14]. Ren et al .2017 conducted a retrospective study, which was assessed whether SSPF +IS is better than SSPF for the treatment thoracolumbar fractures, the inclusion criteria were a single-segment thoracolumbar fracture, no neurological defect and operative decompression, treatment by SSPF / SSPF+IS without fusion. The results demonstrated that pedicle fixation of the fracture did not obtain better recovery of anterior or posterior vertebral heights nor did it improve anterior wedge angle restoration. There was no significant difference between the two surgical groups (SSPF and SSPF+IS) regarding the incidence of broken screws [15]. In our clinical experience, the posterior SSPF without fusion was performed for the treatment patients with AO Type A3 (preoperative LSC score $\leq 6$ points) fractures without neurological impairment. The radiological evaluation of our clinical data revealed that 
$\mathrm{SI}^{\circ}$ and $\mathrm{ABHC} \%$ measurements were significantly decreased after SSPF compared to the preoperative status and maintained through to the long-term follow-up 12 months. Respectively, the VAS pain score was significantly decreased postoperatively and maintained at favourable levels at 12 months follow- up period. The study by La Maida et al. 2016 conducted a comparative analysis of radiological and clinical outcomes of patients with AO Type A3 fractures who underwent MSF/SSPF ( preoperative LSC score $\leq 6$ ) with granular allograft fusion. The results of this study demonstrated that there was no significant difference between MSF /SSPF group patients respecting the $\mathrm{SI}^{\circ}, \mathrm{ABHC} \%$ and VAS score in the long -term follow up; there was no case of the instrumentation failure, or other complications [16]. Unlike of La Maida et al.2016 study in our clinical practice we did not routinely perform posterior or posterolateral fusion for the treatment $\mathrm{AO}$ Type A3 fractures, when facet joint or interspinal ligament was not injured, because of non-fusion method can prevent of adjacent segment degeneration, donor-site morbidity as well as decrease the duration of the surgery and blood loss [17]. Lan et al .2017 performed a systematic review and meta-analysis, the study demonstrated that non-fusion with posterior SSPF obtained radiographic and clinical results like those of posterior SSPF with fusion for thoracolumbar burst fractures [15]. Thus, posterior SSPF can provide safe and promising short- and middle-term results in patients with incomplete thoracolumbar burst fractures (McCormack load-sharing (LSC) score $\leq 6$ ) without neurological deficit who underwent short-segment posterior fixation (SSPF); however, the long-term benefit from treating patients with SSPF further should be analysed [18-20]. The limitation of this study that short- and medium-term clinical and radiological outcomes after SSPF were analysed; the long-term outcomes of this research will be reported in our future publication.

\section{Conclusion}

The results of this study revealed that the conventional SSPF led to significant improvements the radiological and clinical outcomes of patients with AO Type A3 (preoperative LSC score $\leq$ 6 points) fractures without neurological impairment during the

12-month follow-up period after surgery.

\section{References}

1. Spinal Implants and Surgery Devices Market by Technology (Fusion, Spine Biologics, VCF, Decompression, Motion Preservation), Product (Thoracic, Cervical, Interbody, Spine Biologics, Non-Fusion, Stimulators), Type (Open, MIS) - Global Forecast to 2025.

2. VandenBerg J, Cullison K, Fowler SA, Matthew S Parsons, Christopher M McAndrew, et al. (2019) Blunt Thoracolumbar-Spine Trauma Evaluation in the Emergency Department: A Meta-Analysis of Diagnostic Accuracy for History, Physical Examination, and Imaging. J Emerg Med 56(2): 153-165.

3. Spiegl UJ, Josten C, Devitt BM, C-E Heyde (2017) Incomplete burst fractures of the thoracolumbar spine: a review of literature. Eur Spine J 26(12): 3187-3198.

4. Abedi A, Mokkink LB, Zadegan SA, Permsak Paholpak, Koji Tamai, et al.
(2019) Reliability and Validity of the AOSpine Thoracolumbar Injury Classification System: A Systematic Review. Global Spine J 9(2): 231242.

5. Abdusattarov K, Khujanazarov I, Alimov I, Alihodjaeva G (2020) Posterior Long Segment Instrumentation Beyond A Failed Percutaneous Vertebroplasty. J Head Neck Spine Surg 4(2): 555633.

6. Pehlivanoglu T, Akgul T, Bayram S, Emre Meric, Mustafa Ozdemir, et al. (2019) Conservative Versus Operative Treatment of Stable Thoracolumbar Burst Fractures in Neurologically Intact Patients: Is There Any Difference Regarding the Clinical and Radiographic Outcomes? Spine (Phila Pa 1976).

7. Joaquim AF, Patel AA, Schroeder GD, Vaccaro AR (2019) A simplified treatment algorithm for treating thoracic and lumbar spine trauma. J Spinal Cord Med 42(4): 416-422.

8. Abdusattarov K, Khujanazarov I, Alimov I, Alihodjaeva G (2020) Strengths and Shortcomings of Short-Segment Pedicle.Instrumentation for the Treatment Thoracolumbar Burst Fractures American Journal of Biomedical Science \& Research. 8. 189-191.

9. Verheyden AP, Spiegl UJ, Ekkerlein H, Erol Gercek, Stefan Hauck et al. (2018) Treatment of Fractures of the Thoracolumbar Spine: Recommendations of the Spine Section of the German Society for Orthopaedics and Trauma (DGOU). Global Spine Journal 8(2_suppl): 34S-45S.

10. Tan T, Rutges J, Marion T, Augusto Gonzalvo, Joseph Mathew, et al. (2019) Anterior versus posterior approach in traumatic thoracolumbar burst fractures deemed for surgical management: Systematic review and meta-analysis. J Clin Neurosci 70: 189-197.

11. Sun C, Guan G, Liu X, Zhang H, Wang B (2016) Comparison of shortsegment pedicle fixation with versus without inclusion of the fracture level in the treatment of mild thoracolumbar burst fractures. Int J Surg 36(Pt A): 352-357.

12. Aono H, Ishii K, Takenaka S, Hidekazu Tobimatsu, Yukitaka Nagamoto, et al. (2019). Risk factors for a kyphosis recurrence after short-segment temporary posterior fixation for thoracolumbar burst fractures. J Clin Neurosci 66: 138-143.

13. Machino M, Yukawa Y, Ito K, Kanbara S, Kato F (2013) The complement of the load-sharing classification for the thoracolumbar injury classification system in managing thoracolumbar burst fractures. J Orthop Sci 18(1): 81-86.

14. Tan T, Rutges J, Marion T, Augusto Gonzalvo, Joseph Mathew, et al. (2019) Anterior versus posterior approach in traumatic thoracolumbar burst fractures deemed for surgical management: Systematic review and meta-analysis. J Clin Neurosci 70: 189-197.

15. Ren HL, Wang JX, Jiang JM (2018) Is Short Same-Segment Fixation Really Better than Short-Segment Posterior Fixation in the Treatment of Thoracolumbar Fractures? Spine (Phila Pa 1976) 43(21): 14701478.

16. La Maida GA, Luceri F, Ferraro M, Carlo Ruosi, Giuseppe Vincenzo Mineo, et al. (2016) Monosegmental vs bisegmental pedicle fixation for the treatment of thoracolumbar spine fractures. Injury $47 \mathrm{Suppl}$ 4: S35-S43.

17. Lan T, Chen Y, Hu SY, Ao-Lin Li, Xin-Jian Yang, et al. (2017) Is fusion superior to non-fusion for the treatment of thoracolumbar burst fracture? A systematic review and meta-analysis. J Orthop Sci 22(5): 828-833.

18. Toyone T, Ozawa T, Inada Kn, Toshiyuki Shirahata, Ryutaro Shiboi, et al. (2013) Short-segment fixation without fusion for thoracolumbar burst fractures with neurological deficit can preserve thoracolumbar motion without resulting in post-traumatic disc degeneration: a 10year follow-up study. Spine (Phila Pa 1976) 38(17): 1482-1490. 
19. Liu H, Wang H, Liu J, Changqing Li, Yue Zhou, et al. (2019) Biomechanical comparison of posterior intermediate screw fixation techniques with hybrid monoaxial and polyaxial pedicle screws in the treatment of thoracolumbar burst fracture: a finite element study. J Orthop Surg Res 14(1): 122 .
20. Park SR, Na HY, Kim JM, Dong-Chan Eun, Eui-Young Son, et al. (2016) More than 5-Year Follow-up Results of Two-Level and Three-Level Posterior Fixations of Thoracolumbar Burst Fractures with LoadSharing Scores of Seven and Eight Points. Clin Orthop Surg 8(1): 71-77.

\section{Your next submission with Juniper Publishers will reach you the below assets}

- Quality Editorial service

- Swift Peer Review

- Reprints availability

- E-prints Service

- Manuscript Podcast for convenient understanding

- Global attainment for your research

- Manuscript accessibility in different formats ( Pdf, E-pub, Full Text, Audio)

- Unceasing customer service

Track the below URL for one-step submission https://juniperpublishers.com/online-submission.php 